\title{
Dietary $n-3$ fatty acids reduce lymphocyte proliferation in broiler chickens
}

\author{
H. S. Al-Khalifa ${ }^{1}$, C. Rymer ${ }^{1}$, I. Givens ${ }^{1}$ and P. Yaqoob ${ }^{2}$ \\ ${ }^{1}$ Department of Agriculture, The University of Reading, UK and ${ }^{2}$ Department of Food Biosciences, The University of \\ Reading, $U K$
}

There has been interest in the enrichment of poultry meat with long-chain $n-3$ PUFA as a means of increasing their consumption by human subjects. There is some concern that high levels of $n-3$ PUFA may have detrimental effects on immune function in chickens. However, research to date is inconsistent with respect to immunomodulation by $n-3$ PUFA. The aim of this experiment was to determine the effects of dietary $n-3$ PUFA on the proliferation of splenocytes, thymocytes and peripheral blood leucocytes in broiler chickens.

One-day-old male Ross 308 broiler chicks ( $n$ 24) were fed a common starter diet for $21 \mathrm{~d}$. At $21 \mathrm{~d}$, birds were randomly allocated to one of two pens, twelve chicks per pen. Water and feed were provided ad libitum. The broilers were fed for $33 \mathrm{~d}$ on one of two wheatsoyabean meal-based diets. Both diets contained $50 \mathrm{~g} / \mathrm{kg}$ added oil, which was either fish oil (FO) or soya oil. Chickens between 41 and $43 \mathrm{~d}$ of age were sacrified and lymphocytes from freshly collected spleen, thymus and blood were prepared. Concanavalin A (Con A), phytohaemagglutinin (PHA) and Staphylococcus aureus (PANSORBIN)-stimulated proliferation of splenocytes, thymocytes and blood leucocytes was assessed by carboxyfluoroscein succinimidyl ester incorporation using flow cytometry. Results were analysed using CellQuest $\mathrm{TM}$ software of flow cytometry and the WEASEL programme (from Walter \& Eliza Hall Institute).

FO significantly inhibited the splenocyte response to Con A $(10 \mu \mathrm{g} / \mathrm{ml})$, PHA $(75 \mu \mathrm{g} / \mathrm{ml})$, and S. aureus $\left(8 \times 10^{5}\right.$ bacteria per well) compared with the control diet $(P<0.05)$ (see Figure for PHA-stimulated proliferation). FO also inhibited the proliferative response of thymocytes to Con A $(P<0.05)$ and of peripheral blood leucocytes to $S$. aureus $(P<0.05)$.

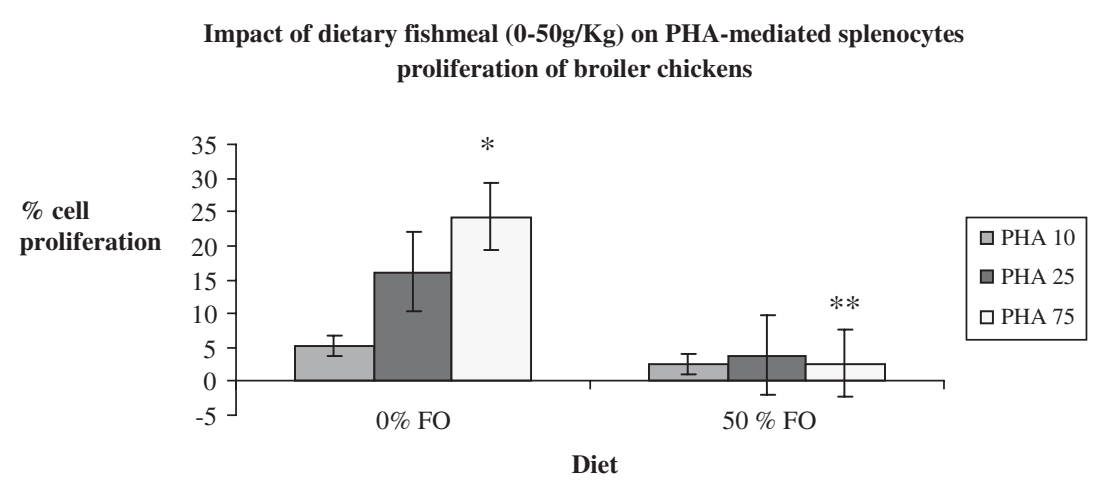

In conclusion, feeding broiler chickens a diet containing $50 \mathrm{~g} / \mathrm{kg}$ FO significantly reduced in vitro proliferation of splenocytes, thymocytes and blood leucocytes. These studies highlight the need for the poultry industry to consider the health status of poultry when poultry meat is being enriched with long chain $n-3$ PUFA.

The authors gratefully acknowledge funding from the Kuwait Institute for Scientific Research. 